\title{
Mechanisms of gap gene expression canalization in the Drosophila blastoderm
}

\author{
Vitaly V Gursky ${ }^{1 *}$, Lena Panok ${ }^{2,3}$, Ekaterina M Myasnikova ${ }^{4}$, Manu ${ }^{3}$, Maria G Samsonova ${ }^{4}$, John Reinitz ${ }^{2,3,5}$ and \\ Alexander M Samsonov ${ }^{1}$
}

\begin{abstract}
Background: Extensive variation in early gap gene expression in the Drosophila blastoderm is reduced over time because of gap gene cross regulation. This phenomenon is a manifestation of canalization, the ability of an organism to produce a consistent phenotype despite variations in genotype or environment. The canalization of gap gene expression can be understood as arising from the actions of attractors in the gap gene dynamical system.
\end{abstract}

Results: In order to better understand the processes of developmental robustness and canalization in the early Drosophila embryo, we investigated the dynamical effects of varying spatial profiles of Bicoid protein concentration on the formation of the expression border of the gap gene hunchback. At several positions on the anteriorposterior axis of the embryo, we analyzed attractors and their basins of attraction in a dynamical model describing expression of four gap genes with the Bicoid concentration profile accounted as a given input in the model equations. This model was tested against a family of Bicoid gradients obtained from individual embryos. These gradients were normalized by two independent methods, which are based on distinct biological hypotheses and provide different magnitudes for Bicoid spatial variability. We showed how the border formation is dictated by the biological initial conditions (the concentration gradient of maternal Hunchback protein) being attracted to specific attracting sets in a local vicinity of the border. Different types of these attracting sets (point attractors or one dimensional attracting manifolds) define several possible mechanisms of border formation. The hunchback border formation is associated with intersection of the spatial gradient of the maternal Hunchback protein and a boundary between the attraction basins of two different point attractors. We demonstrated how the positional variability for hunchback is related to the corresponding variability of the basin boundaries. The observed reduction in variability of the hunchback gene expression can be accounted for by specific geometrical properties of the basin boundaries.

Conclusion: We clarified the mechanisms of gap gene expression canalization in early Drosophila embryos. These mechanisms were specified in the case of hunchback in well defined terms of the dynamical system theory.

\section{Background}

Development is surprisingly robust to environmental stress, intrinsic fluctuations, and genetic variability in populations. These facts, together with the observation that cell type is a discrete rather than continuous property, led C. H. Waddington to propose that developmental processes have innate error-correction properties, which he called "canalization" [1]. Waddington

\footnotetext{
* Correspondence: gursky@math.ioffe.ru

'Theoretical Department, loffe Physical-Technical Institute of the Russian

Academy of Sciences, St. Petersburg, 194021 Russia

Full list of author information is available at the end of the article
}

visualized error correction in terms of an "epigenetic landscape," in which the developmental state of an organism is analogous to a ball rolling down a sloping landscape containing multiple "hills" and "valleys": as development progresses, cells take different paths down this landscape and so adopt different fates. Uncontrolled differentiation does not occur because the hills act as barriers and the state remains near a valley floor. This picture has natural corollaries in terms of genetic variability and evolution. Although the shape of the landscape may alter slightly in the face of genetic variation, the tendency of the system to stay near the valley floor
Ciomed Central

() 2011 Gursky et al; licensee BioMed Central Ltd. This is an Open Access article distributed under the terms of the Creative Commons Attribution License (http://creativecommons.org/licenses/by/2.0), which permits unrestricted use, distribution, and reproduction in any medium, provided the original work is properly cited. 
will buffer the phenotypic consequences of these variations. Even under larger changes over evolutionary time, the tendency of the system to stay close to the valley floors will preserve and buffer developmental pathways in the face of evolutionary change.

We recently demonstrated the existence of canalization at the molecular level in the segment determination system of the fruit fly Drosophila melanogaster [2]. We showed that with respect to the gap gene system, canalization was a consequence of gap gene cross-regulation [3], and furthermore that it was associated with error correction by dynamical attractors [4], a precise mathematical formulation of Waddington's ideas about hills and valleys [5]. That work remains incomplete for reasons involving both broad biological considerations and specific mathematical points. With respect to large scale biological issues, the fact that canalization is under the control of natural selection means that all regulatory systems in an organism are in some sense selected for canalizing properties. In a general evolutionary context, canalization tends to appear as a multigenic trait involving the buffering of underlying genetic variation [6-8]. In a specific example concerning the segmentation system, it was shown that the differing proportional placement of even-skipped stripes in three lines and two species of Drosophila [9] depended on differences in the maternally expressed genes of flies in these lines, rather than the zygotic gap genes, the cross-regulation of which we have shown to ensure proportional spacing [3] because of the alternating arrangement of strongly mutually repressing gap gene expression domains [10].

In comparing our results to those of Lott and coworkers, we note that we considered the canalizing behavior of a specific zygotic component of the segmentation system, while the study of Lott et al. looked at the canalizing behavior of the full set of organismal genes, many subsets of which are presumably engaging in their own form of canalizing behavior. Closing the gap between the genetic control of canalization at the level of populations versus the well defined small networks of genes considered in developmental genetics will require finding the specific genes responsible for population effects as well as taking the complementary step of incorporating additional mechanisms and genes into the well characterized systems arising in a developmental genetics context.

In $[3,4]$, we used the "gene circuit" approach to demonstrate that the reduction in variance of gap gene expression was a consequence of gap gene cross regulation. Gene circuits [11-13] are dynamical models that can reproduce observed gene expression patterns by reconstituting the required set of genetic interactions in silico. Our model accounts for the expression of four mutually interacting gap genes, hunchback (hb), Krüppel
$(\mathrm{Kr})$, giant (gt), and knirps (kni), and takes into account the expression of the genes bicoid (bcd), caudal (cad), and tailless $(t l l)$ as external inputs. Parameter values in the model were calculated by fitting solutions to mean time dependent expression levels from zygotic and maternal/zygotic genes and $b c d$ expression from a single embryo. The resulting model, when run with Bcd gradients from many individual embryos, correctly predicted the variance in position of six gap gene borders. Analysis of model behavior at the numerical level showed that the observed variance was a consequence of gap gene cross regulation [3]. To further elucidate the general nature of the mechanisms controlling variance, we turned off diffusion and analyzed the circuit in individual nuclei using ideas from dynamical systems theory.

This analysis showed that the observed reduction in variation of gap gene expression patterns is a consequence of the action of robust attracting states [4]. The formation of borders of gap gene expression domains could be understood in terms of three qualitative dynamical mechanisms: (1) The movement of attractors; (2) Selection of attractors; (3) Selection of states on a one dimensional attracting manifold. The last of the three mechanisms also causes the domain shifts of the gap genes.

There were two limitations to this analysis. First, the dynamical interactions underlying the observed reduction in variance were elucidated by performing the dynamical analysis on one particular circuit controlled by the median Bcd gradient used for the fit. As a consequence, the variance reduction analysis described in [4] was shifted towards considering only how attractors canalize the variance of initial conditions in the dynamical system, without characterization of Bcd dependence of these attractors. It is possible that other Bcd gradients may entail different dynamical mechanisms of pattern formation. A second limitation is that the individual Bcd gradients used in the analysis had certain systematic scaling errors that exaggerated the variation in threshold location [3,14]. In this paper we extend the dynamical analysis to multiple Bcd gradients, revealing additional dynamical mechanisms which nevertheless work in a coordinated manner to reduce variance. Moreover, we extend the analysis to a system in which the systematic exaggeration of Bcd variance has been removed.

The key idea of this extended analysis is to express gene expression variability in terms of such basic objects of the dynamical systems theory as attractors and attraction basins. The canalization will be explained by specific geometrical properties of these objects. We demonstrate the applicability of this approach using $h b$ border formation as an example. We analyzed how this border forms in terms of the phase portrait of the gap gene dynamical system for various Bcd profiles. We find 
two specific mechanisms responsible for canalization of the variance, both connected to the phase portrait geometry.

\section{Methods}

The ensemble of Bcd concentration profiles

Two sets of spatial profiles of the Bcd concentration were obtained by the numerical processing of raw Bcd data from individual embryos in two different ways. One set was identical to that previously described [3]: the Bcd profiles were retrieved from 89 embryos in the FlyEx database [15-17], with the background removed by basic normalization as described [18]. This method as applied to Bcd profiles assumes that the Bcd profile is exponential and that the background profile is quadratic, a point independently supported by staining in null mutants. One embryo was rejected because of a nonexponential profile [3], thus in total 88 profiles were used in the study. For crosschecking purposes, we applied an alternative normalization method to the same raw Bcd data set as described [14]. The method adjusts both concentration scale and levels of constant background to minimize variance in the ensemble. This way of renormalizing data is useful because it is clear from comparison to in vivo work that our profiles exaggerate variance in Bcd amplitude. However, because there is no independent reason to believe that background should be adjusted to minimize variance, the in situ data processed in this manner should be viewed as a lower limit on embryo to embryo variation of the Bcd profile.

The normalized Bcd profiles were approximated by exponential functions $v^{\mathrm{Bcd}}(x)=A \exp (-l x)$ with $x$ varying along the A-P axis of the embryo. We obtained in this way a set of 88 ( 89 for the alternative normalization method) Bcd parameters $\{A, l\}$ (Additional file 1: Figure S1). The Bcd concentrations in different nuclei at the A$\mathrm{P}$ axis were calculated as $v_{i}^{\mathrm{Bcd}}=v^{\mathrm{Bcd}}\left(x_{i}\right)$, where $x_{i}$ was the position of $i$ th nucleus. We selected a "median" Bcd profile by picking an embryo with parameters $\{A, l\}$ closest to the centroid point in the set of all $\{A, l\}$ values. This median profile was used to fit the gap gene circuit as described [3].

\section{The gap gene circuit}

We modeled the expression of the network of four gap genes $h b, K r, g t$, and $k n i$ with the following equations $[3,4,13,19]$ :

$$
\begin{aligned}
\frac{d v_{i}^{a}}{d t}= & \chi(t) R^{a} g\left(\sum_{b=1}^{N} T^{a b} v_{i}^{b}+m^{a} v_{i}^{\mathrm{Bcd}}+V_{i}^{a}(t)+h^{a}\right)+ \\
& +D^{a}(n)\left[\delta_{i}^{1}\left(v_{i-1}^{a}-v_{i}^{a}\right)+\delta_{i}^{\mathrm{M}}\left(v_{i+1}^{a}-v_{i}^{a}\right)\right]-\lambda^{a} v_{i}^{a},
\end{aligned}
$$

where $v_{i}^{\mathrm{a}}(t)$ is the concentration of protein encoded by gene $a(1 \leq a \leq N, N=4)$ in nucleus $i(1 \leq i \leq M(n))$ along the A-P axis of the embryo. The model incorporates nuclear divisions, number of nuclei $M(n)$ and diffusion coefficients $D^{a}(n)$ depend on the cleavage cycle number $n$. The function $g(u)=\frac{1}{2}\left(1+\frac{u}{\sqrt{1+u^{2}}}\right)$ has a sigmoidal graph and describes regulated scaling for the maximal rate $R^{a}$ synthesis of the ath protein. The argument $u$ of $g$ contains inputs from various transcriptional regulators. The first input is a linear combination of all $v_{i}^{b}$ from the network with parameters $T^{a b}$, which thus quantify the regulatory interactions between the genes. The Bcd concentration profile $v_{i}^{\mathrm{Bcd}}$ is a time invariant maternal input to the system. The term $V_{i}^{a}(t)=E^{a} v_{i}^{\mathrm{Cad}}(t)+F^{a} v_{i}^{\mathrm{Tll}}(t)$ is a given time-dependent external input from the transcription factors Caudal (Cad) and Tailless (Tll). The function $\chi(t)$ equals one during interphase and zero during mitosis, accounting for the fact that synthesis shuts down during this period. Coefficient $\delta_{i}^{1}\left(\delta_{i}^{M}\right)$ equals zero if $i=1(i=M)$ and one otherwise, preventing protein diffusion outside of the spatial domain. Coefficient $\lambda^{a}$ is the rate of protein degradation, and constant $h^{a}$ adjusts the threshold of the regulation function.

The initial conditions in the model consist of a spatial gradient of maternally expressed Hunchback $(\mathrm{Hb})$ protein concentration and zero concentrations of the other three proteins. These conditions correspond to the state of the gap gene system at the start of cleavage cycle 13 . The solutions in the model are biologically meaningful until the end of cleavage cycle $14 \mathrm{~A}$, at which time the midblastula transition occurs and many properties of the embryo change. The nuclei range along the A-P axis within the spatial domain from $35 \%$ to $92 \%$ of the embryo length (EL) where the gap genes express $[3,4,13]$. This spatial domain includes 30 nuclei in cleavage cycle 13 and 58 in cycle $14 \mathrm{~A}$.

All gene expression levels $v_{i}^{a}$ are on a scale of 0-255 chosen to maximize dynamic range in the experimental data without saturation. These levels are called relative concentration units throughout the paper. Time dependent inputs for $v_{i}^{\mathrm{Cad}}(t)$ and $v_{i}^{\mathrm{Tll}}(t)$ were obtained from the data by averaging Cad and Tll expression patterns over individual embryos at various time points, as described in details in earlier work [2]. The background was preliminary removed from the individual Cad and Tll patterns as described [18]. The parameters for the model with Bcd normalized as described [18] were those reported in (See Supplementary Material in [3]). For the alternative normalization procedure, we selected a new median Bcd profile from the renormalized data and fitted the model with that profile to the same time 
dependent averaged gap gene data used previously by either serial or parallel Lam simulated annealing $[11,20]$.

\section{Simplified equations}

We considered a simplified version of the model without diffusion, called the "shorted model" as in previous work [4], keeping the parameter values equal to those found by the fitting procedure in the full model (1). Neglect of the diffusion term in (1) decouples nuclei and reduces $4 \times M$ model equations to $M$ independent systems of four equations, each system corresponding to one nucleus. We investigated only a restricted region of the A-P axis surrounding the posterior border of the anterior $h b$ expression domain. The region ranges from $37 \%$ to $57 \%$ EL, about 11 nuclei in width in cleavage cycle 13 and 22 in cleavage cycle 14A. Tll does not act in this region and can be omitted [4]. We can then analyze shorted equations [4] given by

$$
\frac{d v_{x}^{a}(t)}{d t}=\chi(t) R^{a} g\left(\sum_{b=1}^{N} T^{a b} v_{x}^{b}+m^{a} v^{\mathrm{Bcd}}(x)+E^{a} v^{\mathrm{Cad}}(x, t)+h^{a}\right)-\lambda^{a} v_{x^{\prime}}^{a}
$$

where index $x$ indicates the parametric dependence of solutions. It stems from the dependence of Bcd and Cad protein concentrations on spatial position as determined from experimental data. Such dependence can come directly from experimental data in nuclei, for which $x=i$, or as a continuous real-valued interpolation $x$.

The earliest observable indications of the mid-blastula transition, which include decay of the Bcd gradient, are evident after time class 6 (T6) of cycle 14A, about ten minutes prior to the onset of gastrulation $[2,4]$. For this reason, we represent the time dependence of $v^{\mathrm{Cad}}(x, t)$ from the beginning of cleavage cycle 13 to the end of time class T6. Thereafter we take $v^{\mathrm{Cad}}(x, t)=v^{\mathrm{Cad}}(x, \mathrm{~T} 6)$ and $\chi(t)=1$ for times $t>$ T6. Thus, the shorted equations (2) are nonautonomous until T6 and autonomous later.

\section{Attracting sets, basins of attraction, and hb border positions}

We analyzed the dynamical system (2) for spatial position $x$ from $37 \%-57 \%$ EL region in two mutually complementary directions. We performed a bifurcational analysis in the autonomous version of the system (2), in which $\chi(t) \equiv 1$ and $v^{\mathrm{Cad}}(x, t)$ was replaced by value $v^{\mathrm{Cad}}$ $(x$, T6). The bifurcation structure in the system was studied on the Bcd-Cad plane, which is the plane with coordinates $v^{\mathrm{Bcd}}$ and $v^{\mathrm{Cad}}$ (with $v^{\mathrm{Cad}}$ corresponding to the Cad concentration at $t=\mathrm{T} 6$ ), by means of the AUTO package [21]. In this way, all equilibria and domains of their existence on the Bcd-Cad plane were calculated and all bifurcations separating these domains were elucidated. From another direction, at each spatial position of eleven nuclei in cycle 13 and for each of the
88 Bcd profiles (89 for the alternative normalization method), we calculated basins of attraction for each point attractor. This was done by evaluating the equations until late times when the solution is stabilized, with 10000 random initial conditions uniformly distributed in the biologically relevant subspace of the initial conditions $\Omega=\left\{0 \leq v^{\mathrm{Hb}} \leq 100, v^{\mathrm{Kr}}=v^{\mathrm{Gt}}=v^{\mathrm{Kni}}=0\right\}(\Omega$ is a part of the $\mathrm{Hb}$ axis in the $4 \mathrm{D}$ phase space of the dynamical system). Each point attractor is the asymptotic limit at late times of dynamics starting from the initial conditions which are grouped in a certain part of $\Omega$, and we call this part the basin of attraction for the attractor. To get a more spatially refined picture, we also performed a calculation of attraction basins at thirty spatial positions in the range $37 \%-57 \% \mathrm{EL}$ for the $\mathrm{Bcd}$ ensemble normalized by the basic method.

For each Bcd profile and fixed spatial position $x$, the attraction basin of each attractor can be represented as an interval $\left(c_{1}(x), c_{2}(x)\right)$ on the $\mathrm{Hb}$ axis, as all other protein concentrations are zero in $\Omega$. The values $v^{\mathrm{Hb}}=c_{1}$ and $v^{\mathrm{Hb}}$ $=c_{2}$ are lower and upper boundaries for the basin, respectively. We used linear spatial interpolation for these values in order to study the attraction basin boundaries as continuous functions of $x$ for each Bcd profile. Some attraction basins consist of more than one disjoint interval on the $\mathrm{Hb}$ axis. In this case, the basin boundaries comprise the boundaries of each connected part of the basin.

We calculated approximations for 1D unstable manifolds of saddles $S$ having a single eigenvalue with positive real part by solving the simplified model equations from two initial conditions $S \pm w$, where $w$ is a scaled eigenvector (corresponding to the unstable eigenvalue) of the Jacobian at the saddle.

In order to calculate the $h b$ border positions in solutions exhibited at the onset of gastrulation, we used spatial interpolation of first and third order for solutions in the shorted and full models, respectively. The orders are different for the two models since the solution in the shorted model is less smooth in space than in the full one because of the absent diffusion term, and the use of high order interpolation schemes could lead to artifacts. As a consequence, we computed the border positions in different ways for the two models. The $h b$ border position in the spatially interpolated solution of the shorted model was defined as the point in the spatial domain at which $\mathrm{Hb}$ concentration reached its half-maximal value, and in the full model as the local inflection point.

\section{Results}

We extend the dynamical analysis reported previously for the median Bcd profile [4] to the entire ensemble of Bcd profiles first introduced in [3]. We placed exponential approximations of Bcd profiles from individual embryos in the model (2) with the parameter values 
calculated by Manu et al. (See Tables S1-S2 of Supplementary Information in [3]) and investigated characteristic features of the phase portraits in the model at various spatial positions.

In visualizing the results of our analysis, it is useful to consider two equivalent representations of the spatial information coded in Eqs. (2), either an explicit parameter $x$ denoting A-P positions in the range 37\%-57\% EL or a point on the Bcd-Cad plane (Figure 1). We further use the Bcd-Cad plane to show the bifurcation diagrams and discrete spatial positions for presenting attraction basins.

For the median Bcd profile, the gap gene expression patterns generated by Eqs. (2) from 35\% to 71\% EL occur in the same order and locations as those generated by the full model equations (1). The only exception is that the borders become very sharp and domains tend to be mutually exclusive (See Figure 2 in [4]). Over the full ensemble of Bcd profiles, we found three classes of behavior associated with the qualitatively different expression patterns of genes $K r$ and $g t$ in the anterior vicinity (Figure 2). These classes were visible, but blurred by diffusion, in our previous study of the numerical behavior of the full model (See Figure 3A in [3]).

\section{Attracting sets and various mechanisms of border formation}

For each Bcd gradient, we performed an analysis of how border formation was driven by dynamical attractors.
Because different Bcd profiles lie on different portions of the Bcd-Cad plane, we first characterized which combinations of attractors are present in different parts of this plane by performing a bifurcational analysis (Figure 3 ) of the shorted model (2). We then calculated the basins of attraction for all attractors at a discrete set of eleven positions corresponding to cycle 13 nuclei in the Bcd-Cad plane with initial conditions varied in $\Omega=\{0 \leq$ $\left.v^{\mathrm{Hb}} \leq 100, v^{\mathrm{Kr}}=v^{\mathrm{Gt}}=v^{\mathrm{Kni}}=0\right\}$.

There are four stationary attractors $\left(A_{1}-A_{4}\right)$ in the portion of the Bcd-Cad plane shown in Figure 3. The attractors can be coded with quadruples consisting of 0 , $\mathrm{X}$, or 1 for each attractor component by inspecting whether the corresponding protein concentrations have small, intermediate, or large values at the attractors (Table 1; see the existence domains for attractors corresponding to the alternative normalization method in Additional file 2: Figure S2; examples of spatial dependence of attractors are shown in Additional files 3 and 4: Figures S3 and S4 for the two normalization methods). For example, $A_{3}=0100$ means that $K r$ is highly expressed at this attractor with the other genes staying repressed. $A_{1}$ is the only attractor that continuously changes its code with varying Bcd and Cad concentrations. There are six domains on the Bcd-Cad plane in which various combinations of $A_{1}-A_{4}$ exist (Figure 3 ). Attractor $A_{1}$ exists inside the entire portion of the considered plane, while the other attractors are involved in the bifurcations at the borders of their existence
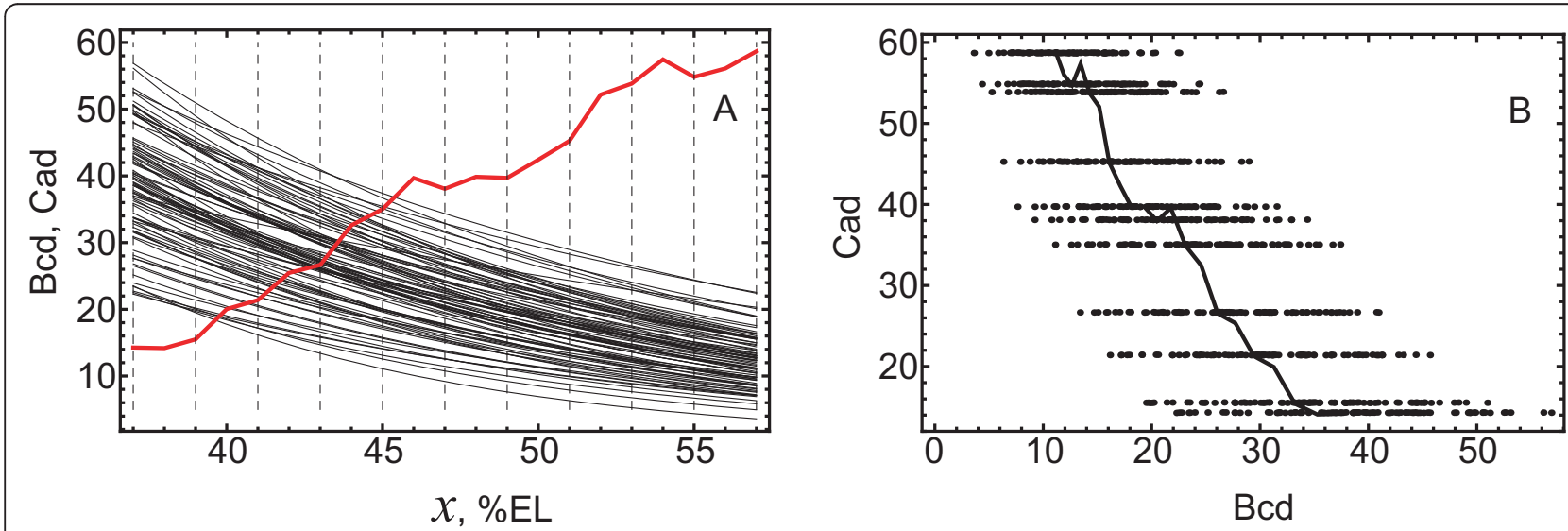

Figure 1 Two representations of the spatial information in the model. (A): The first representation is provided by the explicit use of the spatial position $x$ at the A-P axis of the embryo. The panel shows the Cad spatial profile from time class 6 of cleavage cycle 14A (red line) and exponentially approximated individual Bcd profiles in the ensemble (black lines). The vertical dashed lines indicate positions of eleven nuclei in cleavage cycle 13 and in the given spatial range. (B): The second representation is provided by the use of 2D parameter ( $\left.v^{\mathrm{Bcd}}, v^{\mathrm{Cad}}\right)$, specifying values of Bcd and Cad concentrations in Eqs. (2). This parameter defines a point on the Bcd-Cad plane. The dots in the panel represent the points whose Cad components come from the intersection points between the dashed lines and the Cad profile in (A), and the Bcd components from the intersections of dashed lines with the Bcd profiles in (A). Therefore, these dots describe the actual values of the external input $\left(v^{\mathrm{Bcd}}, v^{\mathrm{Cad}}\right)$ in Eqs. (2) at late times and for the selected eleven spatial positions. The solid line in the panel is the curve $\left(v_{\mathrm{med}}^{\mathrm{Bcd}}(x), v^{\mathrm{Cad}}(x)\right)$ parameterized by $x$ from the spatial range, where $v_{\text {med }}^{\mathrm{Bcd}}(x)$ is the median Bcd profile and $v^{\mathrm{Cad}}(x)$ is the Cad profile from (A), showing how the variation of spatial position $x$ in the model with the fixed Bcd profile is read on the Bcd-Cad plane. 


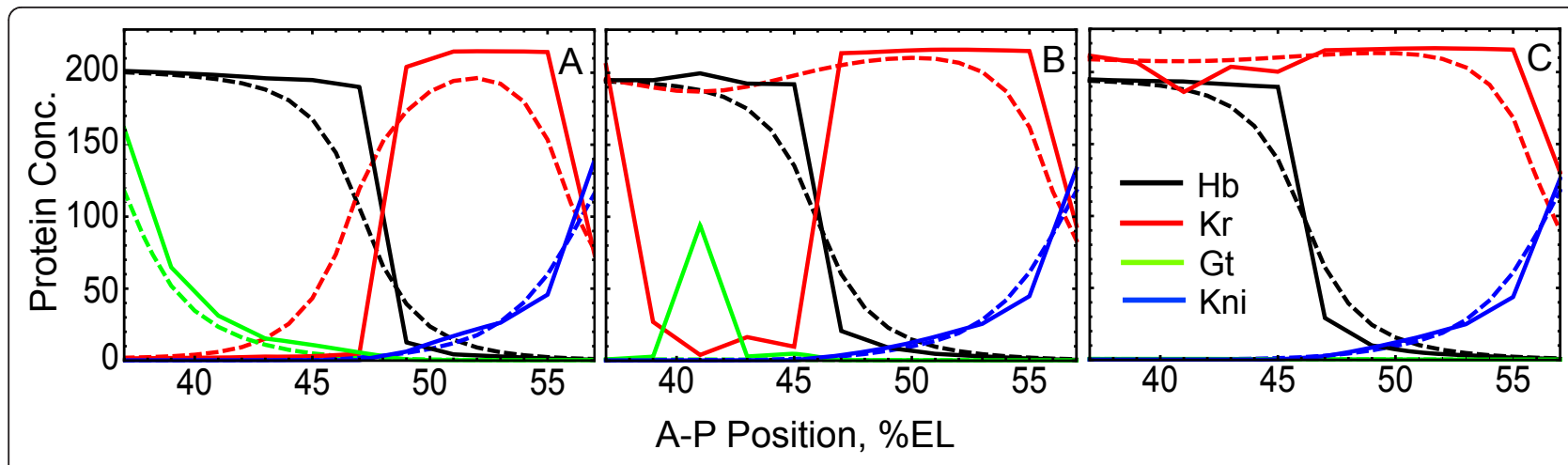

Figure 2 Three classes of solutions. Examples of solutions at time class $6(t=T 6)$ in the full (dashed lines) and simplified (solid lines) models for three different Bcd profiles corresponding to three classes I-III (A-C, correspondingly). The simplified model equations were obtained from the full model equations by neglecting the diffusion term and the influence from TII (see Methods). (A): In class I, all borders are present and in the correct order, as is the case with the median Bcd profile. (B): In class II, the anterior Kr border is missing in the full model but present in the simplified model, typically in association with ectopic anterior expression of $K r$ in place of $g t$, possibly with some ectopic gt expression. (C): In class III, the anterior $\mathrm{Kr}$ border and anterior gt domains are absent in both the full and simplified models.

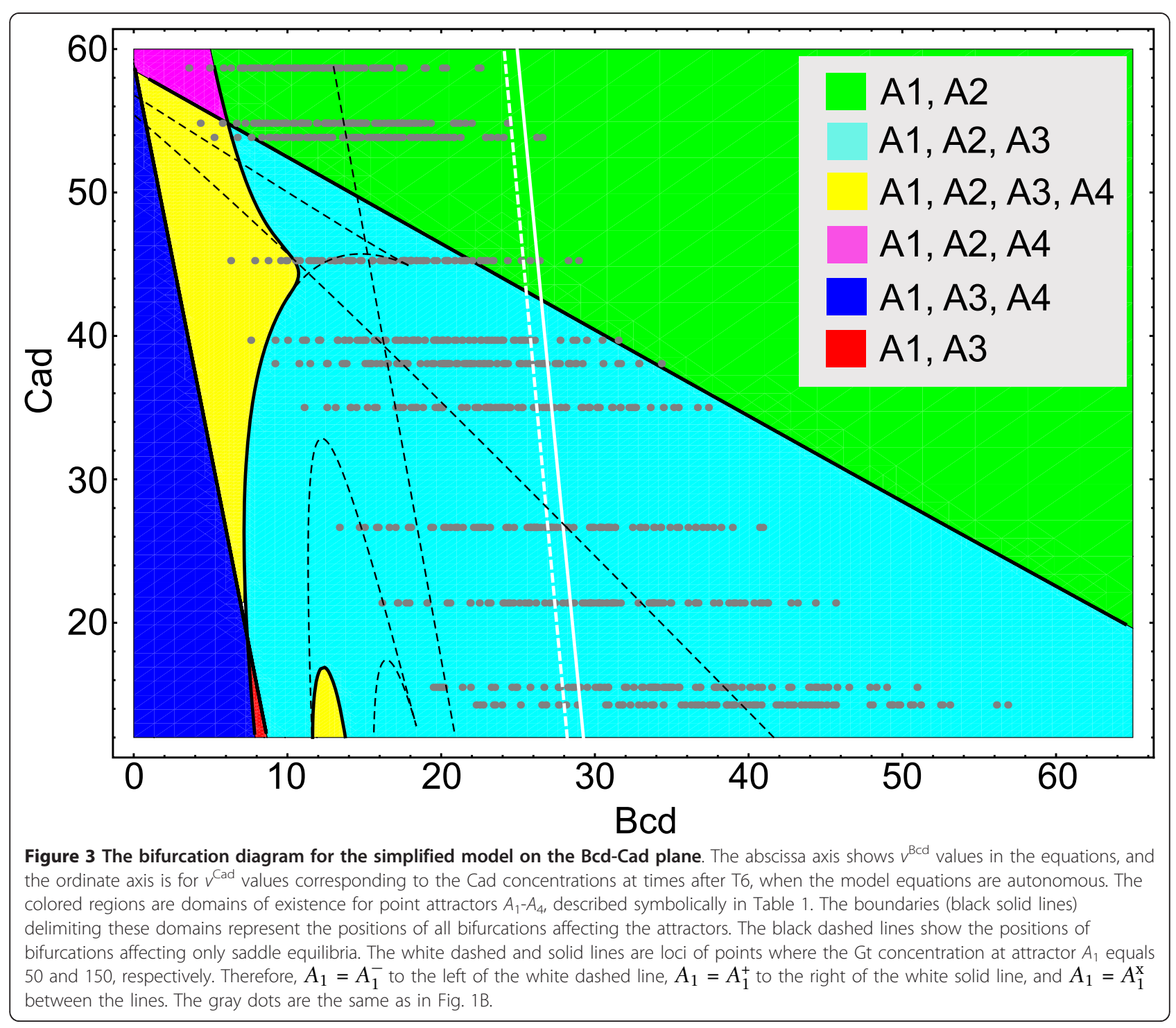


Table 1 The symbolic codes for all attractors in the study calculated for the Bcd and Cad ranges from Figure 3.

\begin{tabular}{ccccc}
\hline & $\boldsymbol{h b}$ & $\boldsymbol{K r}$ & $\boldsymbol{g t}$ & $\boldsymbol{k n i}$ \\
\hline$A_{1}^{-}$ & 1 & 0 & 0 & 0 \\
\hline$A_{1}^{\mathrm{x}}$ & 1 & 0 & $\times$ & 0 \\
\hline$A_{1}^{+}$ & 1 & 0 & 1 & 0 \\
\hline$A_{2}$ & 1 & 1 & 0 & 0 \\
\hline$A_{3}$ & 0 & 1 & 0 & 0 \\
\hline$A_{4}$ & 0 & 0 & 0 & 0 \\
\hline$A_{5}$ & 0 & 1 & 0 & 1 \\
\hline$A_{6}$ & 1 & 0 & 0 & 0 \\
\hline
\end{tabular}

A component of an attractor is assigned the code value ' 1 ' if the concentration of the corresponding protein is larger than 150 relative concentration units, value ' $O$ ' if it is smaller than 50 relative units, and value ' $X$ ' if it is between 50 and 150 (see Methods for description of relative units for protein concentrations). All attractors except $A_{1}$ preserve a single code across the considered ranges for $\mathrm{Bcd}$ and $\mathrm{Cad}$ concentrations. Attractor $A_{1}$ changes its state from $A_{1}=A_{1}^{-}$through $A_{1}=A_{1}^{\mathrm{x}}$ to $A_{1}=A_{1}^{+}$due to continuous increase of the $\mathrm{Gt}$ concentration for this attractor on the Bcd-Cad plane (see the existence domains for these states in Fig. 3 for the basic normalization method and Additional file 2: Figure S2 for the alternative one). All listed attractors exist in the model corresponding to the alternative normalization method, and only $A_{1}-A_{4}$ in the model associated with the basic method.

domains. There are three types of bifurcations found affecting attractors: saddle-node, Hopf, and BogdanovTakens bifurcations (see more detailed description of all bifurcations in Additional file 5: Protocol S1 and Additional file 6: Figure S5). A limit cycle appears at the Hopf bifurcation, which is a nonstationary oscillating attracting state. However, we have not found any oscillatory attractors when the initial conditions are varied in $\Omega$, and, therefore, we exclude this type of attractor from the analysis. The gray dots in Figure 3 show the late time values of the external input in Eqs. (2) used in the calculations of attraction basins for eleven nuclei positions and all of the Bcd profiles. The location of the grey dots on the bifurcation diagram indicates the existence of specific attractors in the phase space for a given nucleus. However, not all of these attractors can be reached under the given biological initial conditions in $\Omega$, as attraction basins of some attractors are disjoint with $\Omega$ (Additional file 7: Figure S6).

As shown elsewhere [4], the dynamics in the model for the median Bcd profile is qualitatively different in parts of the A-P axis which are anterior and posterior to the position of the bifurcation annihilating $A_{3} \cdot$ This position appears as a diagonal line running from the upper left to lower right of Figure 3. The anterior and posterior dynamical regimes are characterized by different types of attracting sets governing the solution dynamics. Solutions at the end of cycle 14A $(t=\tau)$ are very close to point attractors in the anterior regime and to attracting manifolds in the posterior one (examples of these attracting sets are shown in Figure 4). We found that these dynamical regimes are preserved across all the individual Bcd profiles.
To study the dynamical mechanisms of $h b$ border formation, we examined the phase portraits in the shorted model at spatial positions on either side of the border. The mechanism determining the border was found by inspecting which attracting sets govern the solution dynamics from the biological initial conditions in the two nuclei, a $h b$-expressing border nucleus placed just anterior to the $h b$ border position and a $h b$-nonexpressing border nucleus just posterior to that. Border formation is interpreted in these terms as a switch of solution between attracting states in these two nuclei.

We found four qualitatively different mechanisms of $h b$ border formation for Bcd profiles from the ensemble, depending on the type of attracting sets approached by the solutions in the two nuclei by $t=\tau$ (Figure 4 ). For example, Figure 4B shows that for corresponding Bcd profile the border forms by a solution switching from attractor $A_{2}=1100$ in the $h b$-expressing border nucleus to a $h b$-OFF state at the unstable manifold of one of the saddles in the $h b$-nonexpressing border nucleus. Other panels in the figure can be interpreted in a similar way.

There are 66 Bcd profiles associated with the phase portraits which exhibit the attractor-attractor switch mechanism of border formation (Figure 4A) and 20 profiles corresponding to the attractor-manifold switch mechanism (Figure 4B), which make these two mechanisms predominant for the Bcd ensemble. The other mechanisms shown in Figure 4C, D are rare and occur for only one Bcd profile each. The distribution of the four mechanisms over the three solution classes from Figure 2 is summarized in Additional file 8: Table S1. For all Bcd profiles, only attractors $A_{1}, A_{2}$, and $A_{3}$ participate in $h b$ border formation. $A_{4}$ contains a nonempty attraction basin in $\Omega$ for only a few nuclei which lie in a portion of the Bcd-Cad plane that never contains the $\mathrm{Hb}$ border (Additional file 7: Figure S6).

\section{Spatial configuration of attraction basins}

To further investigate how the border formation mechanisms imply the observed $h b$ border variance, we calculated the spatial and Bcd dependence of attraction basin boundaries. We visualize the way that the biological initial conditions choose a basin in different nuclei and try to connect it to the appearance of the $h b$ border in the solution by the time $t=\tau$. We construct graphs combining the initial $\mathrm{Hb}$ profile and the attraction basins for all attractors at different spatial positions, and highlight the range of positional variance for $h b$. Typical examples of these graphs are shown in Figure 5A,B.

For all but one of the Bcd profiles, $h b$ border formation is associated with the transition of the initial $\mathrm{Hb}$ profile from the basin of one point attractor to the basin of another point attractor. This can be seen by comparing the attraction basins of the initial $\mathrm{Hb}$ concentration 


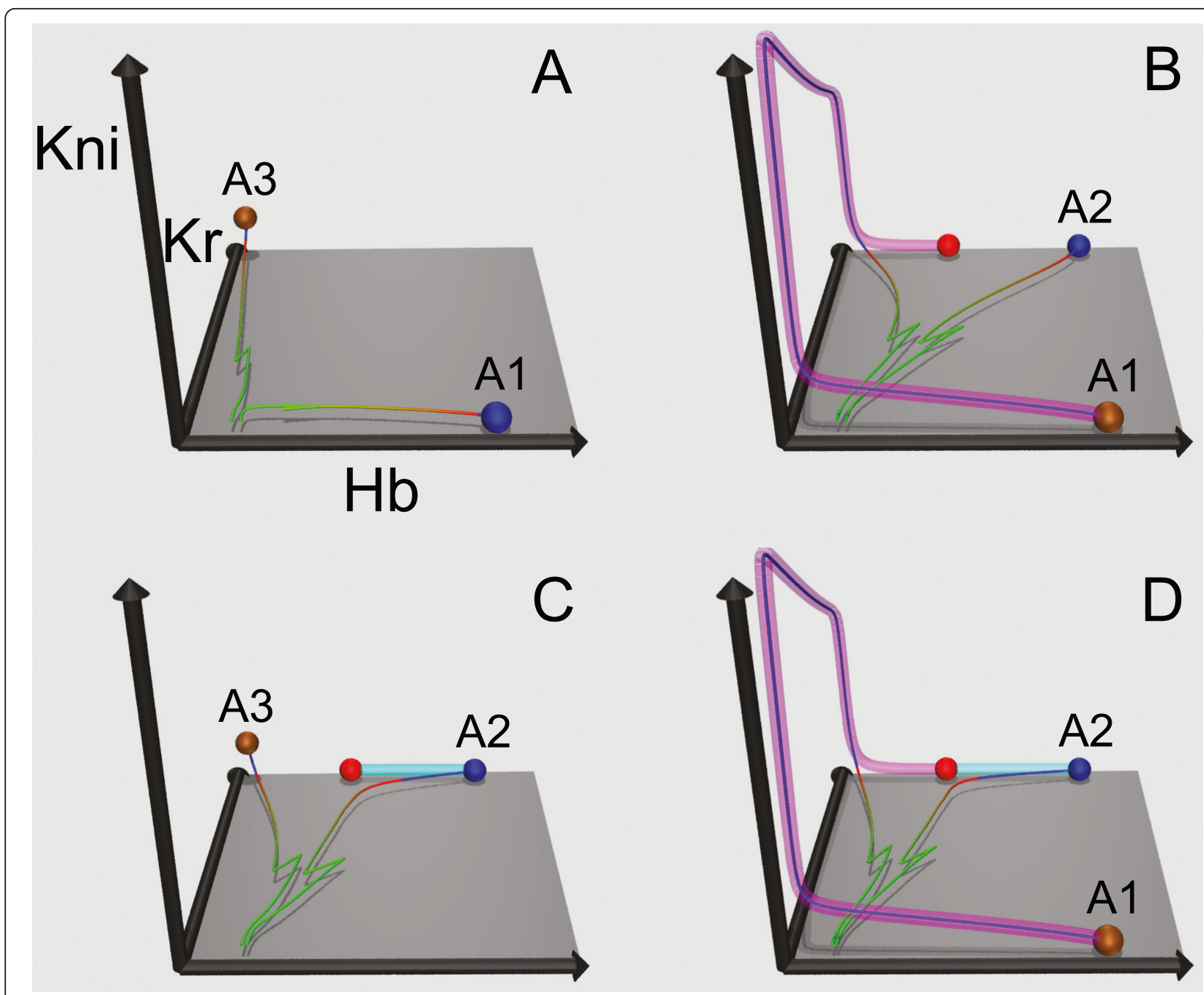

Figure 4 Dynamical mechanisms of hb border formation for various Bcd profiles. Each panel shows the Hb-Kr-Kni projections of the 4D phase portrait fragments from the $h b$-expressing and $h b$-nonexpressing border nuclei simultaneously. The $h b$-expressing and $h b$-nonexpressing border nuclei are the ones placed just anterior and just posterior to the $h b$ border position, respectively. The axes labels for all panels are as for (A) and show the corresponding protein concentrations. The blue balls are the attractors from the hb-expressing border nucleus phase portraits, and the brown ones from the $h b$-nonexpressing nucleus portraits. The red balls are the saddles from the nuclei specified below for each panel. The cyan tubes are branches of the unstable manifolds for the $h b$-expressing border nuclei saddles, and the magenta ones for the $h b$ nonexpressing nuclei saddles. The green trajectories are the solutions for the biological initial conditions from the $h b$-expressing and $h b$ nonexpressing border nuclei. The initial conditions consist of the maternal $\mathrm{Hb}$ concentration $v_{\mathrm{mat}}^{\mathrm{Hb}}$ and are zero for all other proteins. The trajectories gradually turn to red as time approaches the end of cleavage cycle $14 \mathrm{~A}(t=\tau)$ and become blue for later times. (A): The picture is for Bcd profile \#1, with the $h b$-expressing border nucleus at 49\%EL $\left(\left(v^{\mathrm{Bcd}}, v^{\mathrm{Cad}}, v_{\mathrm{mat}}^{\mathrm{Hb}}\right)=(13.7,39.7,29.4)\right)$ and the $h b$-nonexpressing border nucleus at 51\%EL $\left(\left(v^{\mathrm{Bcd}}, v^{\mathrm{Cad}}, v_{\mathrm{mat}}^{\mathrm{Hb}}\right)=(12.1,45.3,23.4)\right)$. (B): Bcd profile \#71; the border nuclei at $49 \% \mathrm{EL}$

$\left(\left(v^{\mathrm{Bcd}}, v^{\mathrm{Cad}}, v_{\mathrm{mat}}^{\mathrm{Hb}}\right)=(26.2,39.7,29.4)\right)$ and 51\%EL $\left(\left(v^{\mathrm{Bcd}}, v^{\mathrm{Cad}}, v_{\mathrm{mat}}^{\mathrm{Hb}}\right)=(24.7,45.3,23.4)\right)$; the red saddle is from the $h b-$ nonexpressing border nucleus. (C): Bcd profile \#6; the border nuclei at 47\%EL $\left(\left(v^{\mathrm{Bcd}}, v^{\mathrm{Cad}}, v_{\mathrm{mat}}^{\mathrm{Hb}}\right)=(23.0,38.1,32.7)\right)$ and $49 \% \mathrm{EL}$ $\left(\left(v^{\mathrm{Bcd}}, v^{\mathrm{Cad}}, v_{\text {mat }}^{\mathrm{Hb}}\right)=(20.7,39.7,29.4)\right)$; the red saddle is from the $h b$-expressing border nucleus. (D): Bcd profile \#37; the border nuclei at $51 \% \mathrm{EL}\left(\left(v^{\mathrm{Bcd}}, v^{\mathrm{Cad}}, v_{\mathrm{mat}}^{\mathrm{Hb}}\right)=(25.8,45.3,23.4)\right)$ and 53\%EL $\left(\left(v^{\mathrm{Bcd}}, v^{\mathrm{Cad}}, v_{\mathrm{mat}}^{\mathrm{Hb}}\right)=(23.5,53.8,20.7)\right)$; the red saddle is from the $h b-$ expressing border nucleus.

values in the two nuclei surrounding the Hb boundary position. For example, the transition in Figure $5 \mathrm{~A}$ is from the basin of $A_{1}$ in the $h b$-expressing border nucleus to the basin of $A_{3}$ in the $h b$-nonexpressing border nucleus (the $A_{1} \rightarrow A_{3}$ transition); the transition in Figure $5 \mathrm{~B}$ is from the basin of $A_{2}$ to the basin of $A_{1}\left(A_{2}\right.$ $\rightarrow A_{1}$ transition). The basin-to-basin transitions can also be detected in Figure 4, where the solutions in the two nuclei go to different point attractors. Bcd profile \#54 provides the only exception from this basin-to-basin transition rule. For this Bcd profile, the solution trajectories in both $h b$-expressing and $h b$-nonexpressing 


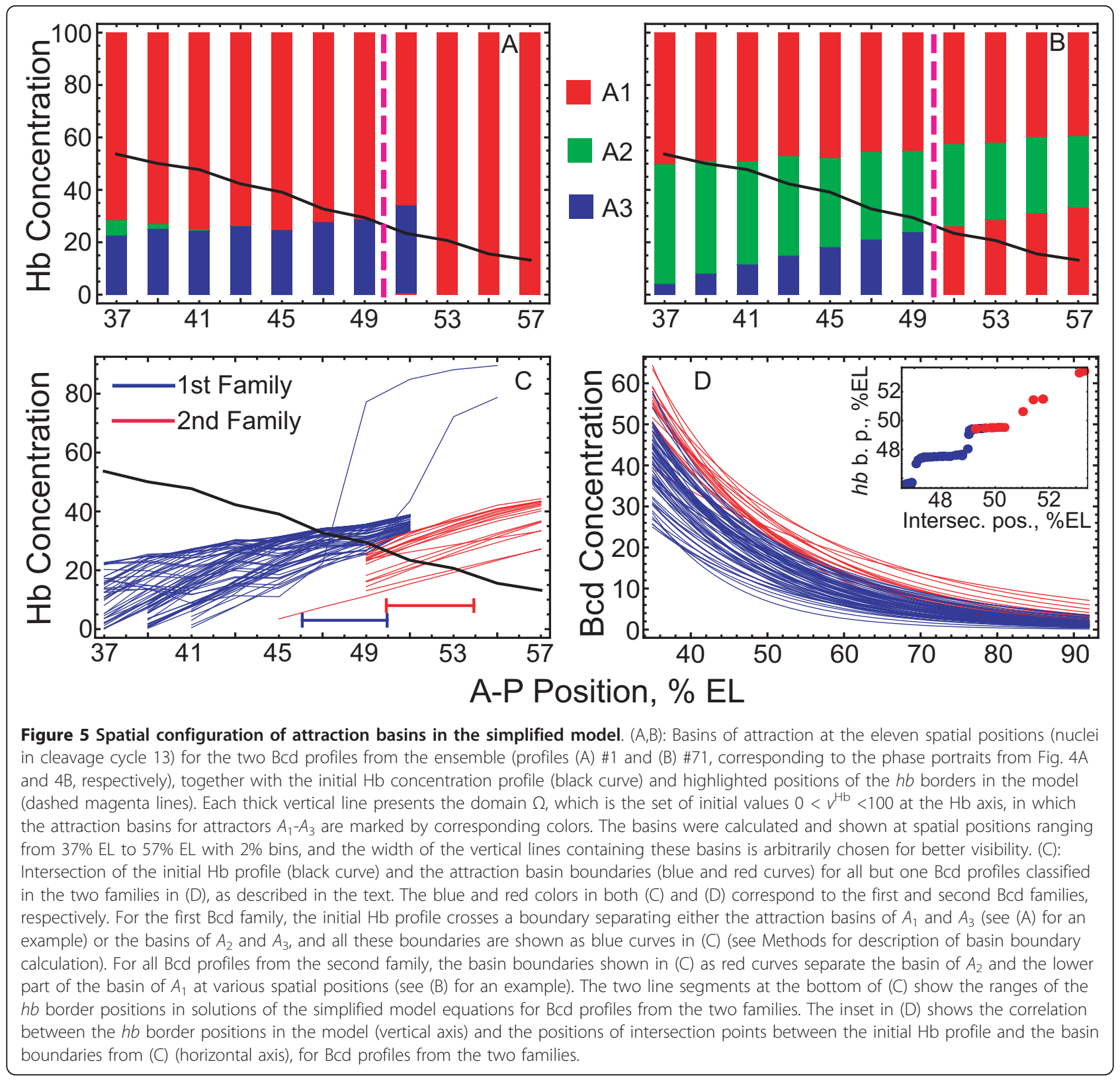

border nuclei eventually end up at the same point attractor $A_{2}$. The $h b$ border forms in this case according to the mechanism from Figure $4 \mathrm{~B}$ (the attractor-manifold switch) with the only difference that the solution trajectory in the $h b$-nonexpressing border nucleus is attracted to a $h b$-OFF state on an unstable manifold which connects a saddle and attractor $A_{2}$ (data not shown). This Bcd profile was excluded from the further analysis. For all cases with the $A_{1} \rightarrow A_{3}$ transition, attractor $A_{1}$ is in the state $A_{1}=A_{1}^{-}$for the $h b$-expressing border nucleus. The $A_{1} \rightarrow A_{3}$ and $A_{2} \rightarrow A_{3}$ transitions are similar in the sense that they both correspond to the switch from an attractor with $h b-\mathrm{ON}$ state to an attractor with $h b$-OFF state. We found $69 \mathrm{Bcd}$ profiles in the ensemble associated with either of these two transitions. We call these profiles Family I in what follows. These transitions can hypothetically correspond to any of the four mechanisms possible for the $h b$ border formation, because the attracting invariant manifolds may also participate in the border formation in this case (see, e.g., Figure 4C). On the other hand, the $A_{2} \rightarrow A_{1}$ transition from Figure $5 \mathrm{~B}$ describes the transition of the initial $\mathrm{Hb}$ concentration between the basins of attractors which both have the $h b$ component in an 'ON' state. The $h b$ border forms in this case by the attracting manifold which provides the necessary $h b$-OFF state at $t=\tau$ (see 
Figure 4B,D). We found a total of $18 \mathrm{Bcd}$ profiles leading to the $A_{2} \rightarrow A_{1}$ transition. We refer to these profiles as Family II. Among these profiles, 16 are associated with the state $A_{1}=A_{1}^{-}$and 2 profiles with the state $A_{1}=A_{1}^{\mathrm{x}}$ for the $h b$-nonexpressing border nucleus. The basin of $A_{3}$ in Figure 5A stops to exist for nuclei located posterior to $51 \% \mathrm{EL}$. The system is in the basin of $A_{1}$ for these positions and approaches a $h b$-OFF state by $t=\tau$ with the help of an attracting manifold. This participation of an attracting manifold in attaining a $h b$-OFF state is the distinctive feature of the aforementioned posterior dynamical regime.

Table 2 shows how the types of initial $\mathrm{Hb}$ transitions between basins are distributed with respect to the border formation mechanisms described above.

\section{Variability of basin boundaries and $\mathrm{hb}$ border positions} The fact that the $h b$ border forms according to the basin-to-basin transition of the initial $\mathrm{Hb}$ profile implies that this profile crosses a boundary between two basins. Therefore, we can relate the border position to the position of the intersection point between the initial $\mathrm{Hb}$ profile and the spatial profile of the basin boundary (see Methods for a description of how this profile is calculated). One can speculate that these two positions are the manifestation of two levels of positional information readout in the gap gene circuit. In this subsection, we compare the positional variability for $h b$ predicted by the model for these two levels. We calculated the spatial profiles of the basin boundaries for all Bcd gradients. The ranges of the border positions corresponding to the two Bcd families are shifted with respect to each other, and the same shift is observed at the level of the basin boundaries (Figure 5C,D). As expected, the positions of the $h b$ border and basin boundary intersection points exhibit strong correlation for both Bcd families (see the inset in Figure 5D). Note that the step-like form of the correlation curve in the figure follows from two factors, the discrete spatial positions used in calculations and the absence of diffusion in the model equations (i.e., the solution of the simplified model more sharply depends on the spatial position).

This correlation means that the intersections between the initial $\mathrm{Hb}$ profile and specific basin boundaries

\section{Table 2 The distribution of Bcd profiles over the four mechanisms of hb border formation and two families described in the text.}

\begin{tabular}{ccccc}
\hline & AA & AM & MA & MM \\
\hline 1st family & 66 & 2 & 1 & 0 \\
\hline 2nd family & 0 & 17 & 0 & 1 \\
\hline
\end{tabular}

The mechanisms are labeled as follows: attractor-attractor switch (AA; Fig. 4A), attractor-manifold switch (AM; Fig. 4B), manifold-attractor switch (MA; Fig. 4C), and manifold-manifold switch (MM; Fig. 4D).
Table 3 Positional variance for $\mathrm{Hb}$ and $\mathrm{Bcd}$.

\begin{tabular}{ccccccc}
\hline & \multicolumn{2}{c}{$\begin{array}{c}\text { First Bcd } \\
\text { family }\end{array}$} & \multicolumn{2}{c}{$\begin{array}{c}\text { Second Bcd } \\
\text { family }\end{array}$} & \multicolumn{2}{c}{$\begin{array}{c}\text { Full Bcd } \\
\text { set }\end{array}$} \\
\cline { 2 - 9 } & f.r. & s.d. & f.r. & s.d. & f.r. & s.d. \\
\hline Intersection positions & 2.9 & 1.0 & 4.0 & 1.2 & 6.6 & 1.5 \\
\hline hb border positions & 3.9 & 1.4 & 4.0 & 1.3 & 7.9 & 1.8 \\
\hline Bcd threshold positions & 13.2 & 3.6 & 6.1 & 1.8 & 19.7 & 4.5 \\
\hline
\end{tabular}

Variability of the $h b$ border positions in the simplified model and the intersection points between the initial $\mathrm{Hb}$ profile and the attraction basin boundaries (from Fig. 5C) for two Bcd families and for the whole Bcd set, in comparison with the positional variability of threshold Bcd concentration. The Bcd threshold value for a family was chosen by picking a Bcd profile corresponding to the mean position of the $h b$ border for that family; then, the threshold value was equal to the value of this Bcd profile at that position. The full range (f.r.) and standard deviations (s.d.) are given in \% of embryo length. The standard deviation for the $h b$ border is $1.3 \%$ in solutions of the full model and $1.0 \%$ in data [3].

encode the $h b$ border positions. As shown in Table 3, the canalization of Bcd variation takes place both at the level of the basin boundaries and at the level of $h b$ border in the simplified model. The variabilities of the intersection points and the $h b$ border positions are approximately the same for each Bcd family and between the families. On the other hand, the Bcd positional variance is significantly different in the two families.

A nonlinear curve of $\mathrm{hb}$ border response to Bcd variation The Bcd dependence of $h b$ border can also be investigated by inspecting the curve representing the response of $h b$ border position to Bcd concentration levels in a vicinity of this position. We studied this response prescribed by the model for Bcd profiles from the ensemble (Figure 6).

The response curve exhibits a specific nonlinear form which is the same in the simplified model (Figure 6A) and in the full one (Figure 6B-C). It persists even if we recalculate the curve by using the normalized individual Bcd profiles instead of their exponential approximations (Additional file 9: Figure S7), indicating that the results are independent of the nuclear noise present in the non-approximated Bcd data.

The nonlinear form of the curve is the result of the reaction to Bcd variation from the whole gap gene network. As a consequence, we have a linearly increasing response curve when gap gene cross regulation of $h b$ is removed from the full model equations (Figure 6C). In that case, the gradually increasing amplitude (or a mass) of Bcd profile across the ensemble results in gradual posterior shift of the $h b$ border (Figure 6E). This happens because Bcd is an activator of $h b$ and the spatial profiles of both Bcd and $\mathrm{Hb}$ concentrations are monotonously decreasing in the vicinity of $h b$ border position. In contrast, there can be reversed (anterior) shifts of the $h b$ border for some range of Bcd concentration in the presence of gap gene cross regulation (Figure 6D). 


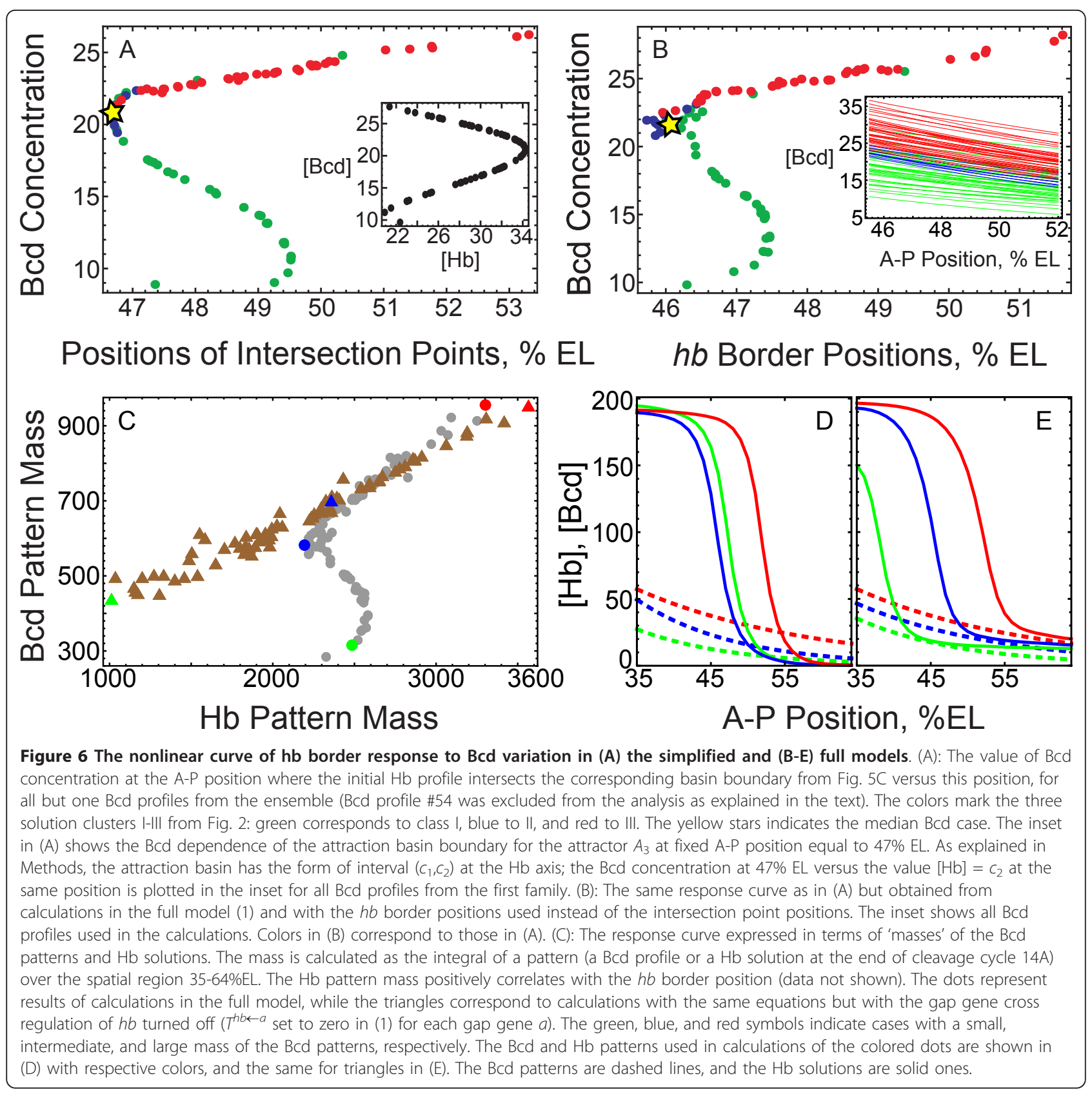

The inset in Figure 6A shows an example for one spatial position of how the attraction basin boundary depends on Bcd concentration. This dependence locally represents the response curve at the level of attraction basins in the model. The specific form of the curve in the inset underlies the nonlinear form of the response curve for the intersection points and border positions.

\section{Results for the alternative Bcd normalization method}

As the Bcd variance can be exaggerated in the data normalized by the basic method, we considered the same ensemble of Bcd profiles but normalized by the alternative artificial normalization method, which minimizes variance in the ensemble (see Methods). We studied the model on the newly normalized Bcd profiles in order to crosscheck our results. The corresponding parameters $A$ and $l$ of exponential approximation for the Bcd profiles are shown in Additional file 1: Figure S1. We calculated a new set of parameter values (Additional file 10: Table S2) in the full model (1) with new median Bcd profile by fitting to the same gap gene expression data that were used previously $[3,4]$. Main qualitative features of these parameter values and corresponding solution of the simplified equations (2) 
(Additional file 11: Figure S8) are very close to those in the above study $[3,4]$.

Due to the specific statement of the alternative normalization method, the Bcd positional variance in the ensemble became much lower: the standard deviation $\sigma$ $=1.2 \% \mathrm{EL}$. The corresponding positional variance for $h b$ in the full model equals to $\sigma=0.5 \% \mathrm{EL}$.

We calculated a new bifurcation diagram on the BcdCad plane for the simplified model (Additional files 2, 12, 13: Figures S2, S9, and Protocol S2). There are two new attractors, $A_{5}=0101$ and $A_{6}=1000$, in addition to attractors $A_{1}-A_{4}$ which are analogs of the corresponding attractors to the above study. Attractor $A_{6}$ exists in a very narrow domain on the Bcd-Cad plane, where it coexists with attractor $A_{1}$ at the state $A_{1}=A_{1}^{\mathrm{x}}$ (Additional file 2: Figure S2). Thus, the attractor 1000 is unique in this domain. The calculations of attraction basins at discrete spatial positions did not detect $A_{4}$, since the newly normalized Bcd concentrations never entered the existence domain for this attractor (Additional file 14: Figure S10). As in the above study, only attractors $A_{1}-A_{3}$ are important for the process of $h b$ border formation. The spatial dependence of these attractors is mostly similar to the previous results, except that the bifurcations changed their positions at the A-P axis (see, e.g., the case of the median Bcd profile in Additional file 4: Figure S4, in comparison with previously discussed Additional file 3: Figure S3). Despite the difference in the bifurcation structure, new calculations also revealed the existence of an attracting invariant manifold governing the dynamics in a posterior spatial region, which is the analog of the previously found manifold [4]. This manifold has similar geometrical properties, reflecting the fact that it is responsible for the observed anterior shifts of the posterior gap domains taking place in cycle 14A [4].

Three of the four mechanisms shown in Figure 4 exist for the new parameter values and for various newly normalized Bcd profiles: the attractor-attractor (Figure 4A), manifold-attractor (Figure 4C), and manifold-manifold (Figure 4D) switches. Analyzing how the initial Hb profile chooses the attraction basin in the nuclei neighboring the $h b$ border, we detected 85 Bcd profiles leading to the $A_{1} \rightarrow A_{3}$ transition, $2 \mathrm{Bcd}$ profiles leading to the $A_{2} \rightarrow A_{3}$ transition, and 2 profiles which were not associated with the change of basins across these nuclei (they corresponded to the switch between different states on the unstable manifold of a saddle) (see Additional file 15: Table S3 for a distribution of these cases with respect to the solution classes I-III). The model demonstrates a picture of the initial $\mathrm{Hb}$ profile crossing the attraction basin boundaries similar to the first family case in Figure $5 \mathrm{C}$, but with a smaller variance (Additional file 16: Figure S11). The Bcd response curve for the $h b$ border in the model with the new parameter values is also not linear (Additional file 17: Figure S12).

\section{Discussion}

\section{Mechanisms of border formation}

We presented the dynamical analysis of the simplified model of the gap gene network on the ensemble of early Drosophila embryos. The main goal was to decode the mechanistic basis of the gap gene border formation and stability under the Bcd morphogen variance. The $h b$ border formation mechanisms were described in terms of attracting sets and their attraction basins calculated in the nuclei surrounding the border position.

The results reveal that the border formation can be associated with the event of intersection between a boundary separating the attraction basins of two different point attractors and the initial $\mathrm{Hb}$ profile presenting the input from the maternally expressed $h b$ gene. Attracting sets of another type, the unstable manifolds of saddle equilibria, actively participate in the adjustment of the border position. They do so by attracting the solution trajectories in the nuclei surrounding this position. The model predicts that these attracting manifolds can be involved in the border formation for some Bcd profiles.

The $h b$ border correctly forms in the model by the onset of gastrulation for all individual Bcd profiles. For about a half of these profiles, however, the $\mathrm{Kr}$ and Gt patterns in the solutions exhibit defects in the anterior part of the spatial domain (solution classes II and III). It turns out that the $h b$ border formation mechanism involving the attracting manifolds is mostly associated with these cases. This may lead to the conclusion about restricted applicability of this mechanism in the case of $h b$ expression. However, this mechanism exists and plays an important role for the gap domain borders in a posterior part of the embryo, where the domains form and vary in time under the control of an unstable manifold [4]. To analyze canalization for the posterior borders, the variation for external inputs from Cad and Tll should be taken into account, where these transcription factors are among the key regulators, and a modified model should be considered including an input from the terminal gene huckebein [22].

\section{Mechanisms of canalization}

As previously reported, the model exhibits a significant filtration (canalization) of the Bcd positional variability at the level of $h b$ border formation [2-4]. Our results show how this filtration stems from the stable behavior of the attraction basin boundaries. It was shown in $[3,10]$ that the mutual regulatory repression between the gap genes accounts for the observed variance reduction, thus presenting a buffering mechanism for canalization 
$[23,24]$. We translated this buffering mechanism to the level of attractors and their attraction basins. As the $h b$ border position is well encoded by the intersection between the initial $\mathrm{Hb}$ profile and corresponding attraction basin boundaries, the stability of $h b$ border predicted by the model can be explained by inspecting the geometrical properties of these attraction basins.

From this inspection, we can elucidate the following two mechanisms responsible for the observed robustness. First, the initial $\mathrm{Hb}$ profile is a monotonously decreasing function of A-P position, while the basin boundary to be crossed is a monotonously increasing one (Figure 5), i.e., these curves have opposite dependencies on the A-P position. This purely geometrical fact evidently prescribes a smaller variation of the intersection point when the basin boundary is changing due to the variance of Bcd concentration, as opposed to the case if the curves would jointly rise or jointly fall along the A-P axis (we illustrated this mechanism of canalization in Additional file 18: Figure S13).

The second mechanism is associated with the specific nonlinear form of the response curve from Figure 6 . The gap gene cross regulation of $h b$ bends the response line exhibited in absence of this regulation (Figure 6C). This bending effectively reduces the $\mathrm{Hb}$ positional variance by about half. In terms of attractors, this bending is controlled by the fact that a basin boundary responsible for the $h b$ border formation does not change monotonously, but oscillate in the state space with the changing Bcd profile.

The results show that the full range of the $h b$ positional variance is broken down into two almost equal parts, the anterior and posterior ones (see the line segment in Figure 5C). These parts are associated with two families of the Bcd individual profiles (Family I and Family II, respectively) and two different mechanisms of $h b$ border formation. The Bcd profiles from Family I lead to the $h b$ border formation as a switch from a $h b$ $\mathrm{ON}$ attractor in a $h b$-expressing nucleus to a $h b$-OFF attractor in a $h b$-nonexpressing nucleus, while for Family II the border forms with the help of an attracting invariant manifold in a $h b$-nonexpressing nucleus. Since the difference between the two families is in the amplitude of the Bcd profiles, we conclude that Bcd profiles of high amplitude canalize by a dynamical mechanism different from those of lower amplitude. Each dynamical mechanism provides only half of the full variance for the $h b$ border, but in two adjacent spatial domains. Therefore, the change of the dynamical mechanism that happens with rising Bcd amplitude effectively doubles the variance.

The $h b$ border positions from the more posterior range are placed posterior to the spatial position of a bifurcation annihilating attractor $A_{3}$. This bifurcation position delimits the anterior and posterior dynamical regimes in the model, as described previously [4]. Therefore, the Bcd profiles from the second family shift the $h b$ border to the posterior dynamical regime, which is characterized by an active role of an attracting invariant manifold in the pattern formation.

The results indicate that the posterior range of $h b$ positional variation is almost equal to the anterior one only due to smaller variation of the Bcd profiles in Family II compared to Family I. This suggests that the solutions in the anterior and posterior dynamical regimes have quite different sensitivity rates to variation of the Bcd concentration. For Family I, the standard deviation for the $h b$ border position is 2.6 times less than for the Bcd threshold position, while it is only 1.4 times less in the case of Family II. This difference can be explained by an observation that Bcd profiles of higher amplitude correspond to the linear part of the response curve from Figure 6, and this is a consequence of specific regulatory interactions in the gap gene circuit as explained further.

We have used the model (1) to study the canalization mechanisms based on the assessment that the model provides one of the best spatio-temporal precision for the description of gap gene expression [25]. This model is an approximation to a more general model of gene regulation, which should be grounded on the statisticalmechanical formalism. One possible limitation is the linear approximation for the argument of the nonlinear regulation function $g$. The canalization mechanisms described in terms of attractors and attraction basins generally depend on the structure of the model that predicts these attracting states. Therefore, an important direction for future investigations should be verification of the proposed mechanisms in a phase space of a more general model.

\section{Response curve}

The nonlinear nature of the Bcd readout by the gap gene circuit is clearly represented in a specific nonlinear form of the response curve showing the Bcd dependence of the $h b$ border position in the model. The nonlinear part of the curve can be explained by the regulatory actions on $h b$ from the other gap genes. In particular, a regulatory analysis in the full model revealed that the regulatory interactions between $h b, g t$, and $K r$ underlie the folding part of the response curve (Additional file 19: Protocol S3). The gap gene cross-regulation also participate in the linear parts of the response curve by tuning the incline of these parts.

It was previously pointed out that the $g t$ and $\mathrm{Kr}$ expression borders in the anterior part of the A-P axis show large variation in the model in response to $\mathrm{Bcd}$ variation because the model is missing some regulators 
in this part [3]. For example, these $g t$ and $K r$ borders are absent in the solutions from class III. This fact raises doubts on the specific folding part that the response curve exhibits in the middle range of the Bcd concentration values. On the other hand, the folding part exists only for the Bcd profiles associated with the solutions from class I, with all expression borders formed correctly, which means that an essential portion of the artificial variation of the $g t$ and $K r$ borders can be excluded from the consideration without affecting the folding form of the curve.

\section{Comparison of results for two normalization methods}

We investigated the model on the ensemble of Bcd profiles normalized by the alternative method, which provided lower Bcd variance [14]. One used this method as an artificial limit case, in which we dealt with the ensemble possessing minimal Bcd variance, and applied it for the crosschecking purposes.

We have not found any essential discrepancy in the mechanisms of $h b$ border formation and canalization for the two normalization methods. A distinct bifurcation structure in the model with the new parameter values does not lead to changes in the solutions during the biologically important time. The model preserves an attracting invariant manifold related to the posterior dynamical regime. The same border formation mechanisms appear except the one associated with the attractor-manifold transition. It is important that, even though the second family of Bcd profiles does not appear in the alternative normalization case, the invariant manifolds still play their role in adjusting the border position. The model also demonstrates an essentially nonlinear response curve for the $h b$ border. Therefore, our conclusions formulated above are robust with respect to the choice of the normalization method, and, in more general terms, they should be valid for different estimates of the actual Bcd variance.

This correspondence can be explained by the fact that the parameters $A$ and $l$ obtained for the alternatively normalized Bcd profiles form a subset in similar parameters obtained in the case of the basic normalization method (see Additional file 1: Figure S1). Roughly speaking, we can associate the alternatively normalized Bcd profiles with Family I. In particular, this means that the Bcd data rescaled according to the alternative algorithm support the conclusion formulated above about different dynamical mechanisms of canalization for Bcd profiles of different amplitude.

There is an important issue concerning the comparison of the Bcd variance filtration rates. The calculations reveal that, for the basic normalization method, the $\mathrm{Hb}$ positional variation of $1.3 \% \mathrm{EL}$ in the model output follows from the $\mathrm{Bcd}$ positional variation of $4.5 \% \mathrm{EL}$, thus implying that more than $70 \%$ of the positional variance has been filtrated. The same calculations for the alternative normalization method give the filtration rate of approximately $60 \%$. Therefore, the filtration still happens in the model even if we normalize Bcd profiles according to the precisionist hypothesis [14]. This result is quite expected since the reported dynamical mechanisms underlying the processing of the Bcd variation in the model are valid irrespective of the absolute variation range. Whatever actual variation the $\mathrm{Bcd}$ morphogen exhibits, the nonlinear model response translates it to a smaller variation of the target gene patterns.

\section{Conclusions}

The formation of $h b$ border is coded by the intersection between the maternal $\mathrm{Hb}$ gradient and a boundary between attraction basins in the gap gene dynamical system. Small positional variance for $h b$ border can be explained by the geometrical properties of this basin boundary and its nonmonotonic dependence on the Bcd concentration. Main features of the phase portraits underlying the canalization mechanisms do not depend on the normalization method for Bcd.

\section{Additional material}

Additional file 1: Parameters $A$ and $I$ of exponential approximation of individual Bcd profiles for two normalization methods.

Additional file 2: The bifurcation diagram for the new parameter values.

Additional file 3: The spatial dependence of attractors in the shorted model for the median Bcd profile.

Additional file 4: The spatial dependence of attractors in the shorted model with the new parameter values and for the median Bcd profile.

Additional file 5: Detailed description of the bifurcations in the model (the text contains reference to Figure S5).

Additional file 6: The bifurcation diagram with more details.

Additional file 7: The existence domains on the Bcd-Cad plane for attractors $A_{1}-A_{4}$ following from calculations at discrete spatial positions.

Additional file 8: The distribution of Bcd profiles over the four mechanisms of $h b$ border formation and over solution classes I-III.

Additional file 9: The response curve for the normalized individual Bcd profiles instead of their exponential approximations.

Additional file 10: New parameter values in the model obtained by optimization with a median Bcd profile from the Bcd data normalized by the alternative method.

Additional file 11: The solutions of the full model equations and their simplified version for the new parameter values.

Additional file 12: The bifurcation diagram for the new parameter values with more details.

Additional file 13: Detailed description of the bifurcations for the new parameter values (the text contains reference to Figure S9).

Additional file 14: The existence domains on the Bcd-Cad plane for attractors $A_{1}-A_{6}$ in the model with the new parameter values following from calculations at eleven spatial positions. 
Additional file 15: Classification results for the Bcd profiles in the case of the alternative normalization method.

Additional file 16: The spatial configuration of attraction basins in the model with the new parameter values.

Additional file 17: The response curve for the new parameter values.

Additional file 18: Schematic illustration of the first canalization mechanism

Additional file 19: The regulatory analysis of the response curve (the Protocol contains Figures S14-S17)

\section{Acknowledgements}

The study was supported by NIH Grant RR07801, CRDF GAP award RUB11578-ST-05, and RFBR grants 08-01-00315a, 08-04-00712a, 10-01-00627a, 1101-00573a, and 11-04-01162a. We thank Konstantin N. Kozlov for the help with the parameter optimization code and Johannes Jaeger for valuable feedback on the manuscript. The optimization calculations were partly performed at the Joint Supercomputer Center of the Russian Academy of Sciences.

\section{Author details}

'Theoretical Department, loffe Physical-Technical Institute of the Russian Academy of Sciences, St. Petersburg, 194021 Russia. ${ }^{2}$ Department of Applied Mathematics and Statistics, and Center for Developmental Genetics, Stony Brook University, Stony Brook, NY 11794-3600, USA. ${ }^{3}$ Chicago Center for Systems Biology, and Department of Ecology and Evolution, The University of Chicago, Chicago, IL 60637, USA. " Department of Computational Biology, Center for Advanced Studies, St. Petersburg State Polytechnical University, St. Petersburg, 195259 Russia. ${ }^{5}$ Department of Statistics, and Department of Molecular Genetics and Cell Biology, The University of Chicago, Chicago, IL 60637, USA.

\section{Authors' contributions}

WG, $M$, and JR conceived and designed the course of the study. WG performed calculations of attractors and attraction basins, model optimization, and analysis of data and results. LP carried out the bifurcational analysis and calculated the phase portraits. EMM applied alternative normalization to the Bcd data and analyzed the data. MGS and JR performed biological analysis of the results. WG and LP made the figures. AMS, MGS, and JR supervised the work. WG, LP, JR, MGS, and AMS wrote the paper. All authors read and approved the final manuscript.

Received: 2 February 2011 Accepted: 28 July 2011

Published: 28 July 2011

\section{References}

1. Waddington $\mathrm{CH}$ : Canalization of development and the inheritance of acquired characters. Nature 1942, 150:563-565.

2. Surkova S, Kosman D, Kozlov K, Manu, Myasnikova E, Samsonova A, Spirov A, Vanario-Alonso CE, Samsonova M, Reinitz J: Characterization of the Drosophila Segment Determination Morphome. Developmental Biology 2008, 313(2):844-862.

3. Manu, Surkova S, Spirov AV, Gursky V, Janssens H, Kim A, Radulescu O, Vanario-Alonso CE, Sharp DH, Samsonova M, Reinitz J: Canalization of gene expression in the Drosophila blastoderm by gap gene cross regulation. PLOS Biology 2009, 7:e1000049.

4. Manu, Surkova S, Spirov AV, Gursky V, Janssens H, Kim A, Radulescu O, Vanario-Alonso CE, Sharp DH, Samsonova M, Reinitz J: Canalization of gene expression and domain shifts in the Drosophila blastoderm by dynamical attractors. PLoS Computational Biology 2008, 5:e1000303.

5. Thom R: Topological Models in Biology. Topology 1969, 8:313-335.

6. Gibson G, Wagner G: Canalization in evolutionary genetics: a stabilizing theory? BioEssays 2000, 22:372-380.

7. Hansen TF: The Evolution of Genetic Architecture. The Annual Review of Ecology, Evolution, and Systematics 2006, 37:123-157.

8. Meiklejohn CD, Hartl DL: A single mode of canalization. Trends in Ecology and Evolution 2002, 17(10):468-473.
9. Lott S, Kreitman M, Palsson A, Alekseeva E, Ludwig M: Canalization of segmentation and its evolution in Drosophila. Proceedings of the National Academy of Sciences USA 2007, 104:10926-10931.

10. Vakulenko S, Manu, Reinitz J, Radulescu O: Having the right proportions: Interacting interfaces ensure robust spatial patterning. Physical Review Letters 2009, 103:168102.

11. Reinitz J, Sharp DH: Mechanism of eve stripe formation. Mechanisms of Development 1995, 49:133-158.

12. Reinitz J, Sharp DH: Gene Circuits and Their Uses. In Integrative Approaches to Molecular Biology. Edited by: Collado J, Magasanik B, Smith T. Cambridge, Massachusetts, USA: MIT Press; 1996:253-272.

13. Jaeger J, Surkova S, Blagov M, Janssens H, Kosman D, Kozlov KN, Manu, Myasnikova E, Vanario-Alonso CE, Samsonova M, Sharp DH, Reinitz J: Dynamic control of positional information in the early Drosophila embryo. Nature 2004, 430:368-371.

14. Gregor T, Tank DW, Wieschaus EF, Bialek W: Probing the limits to positional information. Cell 2007, 130:153-164.

15. Poustelnikova E, Pisarev A, Blagov M, Samsonova M, Reinitz J: FlyEx Database. 2005 [http://urchin.spbcas.ru/flyex].

16. Poustelnikova E, Pisarev A, Blagov M, Samsonova M, Reinitz J: A database for management of gene expression data in situ. Bioinformatics 2004, 20:2212-2221.

17. Pisarev A, Poustelnikova E, Samsonova M, Reinitz J: FlyEx, the quantitative atlas on segmentation gene expression at cellular resolution. Nucleic Acids Research 2008, 37:D560-D566[http://nar.oxfordjournals.org/cgi/content/ abstract/gkn717v1].

18. Myasnikova E, Samsonova M, Kosman D, Reinitz J: Removal of background signal from in situ data on the expression of segmentation genes in Drosophila. Development, Genes and Evolution 2005, 215:320-326.

19. Mjolsness E, Sharp DH, Reinitz J: A connectionist model of development. The Journal of Theoretical Biology 1991, 152:429-453.

20. Chu KW, Deng Y, Reinitz J: Parallel simulated annealing by mixing of states. The Journal of Computational Physics 1999, 148:646-662.

21. Doedel E, et al: AUTO: Software for continuation and bifurcation problems in ordinary differential equations.[http://indy.cs.concordia.ca/ auto].

22. Ashyraliyev M, Siggens K, Janssens H, Blom J, Akam M, Jaeger J: Gene Circuit Analysis of the Terminal Gap Gene huckebein. PLOS Computational Biology 2009, 5:e1000548.

23. Waddington $\mathrm{CH}$ : Organisers and Genes Cambridge, U.K.: Cambridge University Press; 1940.

24. Waddington $\mathrm{CH}$ : The Strategy of Genes London: George Allen \& Unwin; 1957.

25. Jaeger J: Modelling the Drosophila embryo. Molecular BioSystems 2009 5:1549-1568.

doi:10.1186/1752-0509-5-118

Cite this article as: Gursky et al:: Mechanisms of gap gene expression canalization in the Drosophila blastoderm. BMC Systems Biology 2011 5:118.

\section{Submit your next manuscript to BioMed Central and take full advantage of:}

- Convenient online submission

- Thorough peer review

- No space constraints or color figure charges

- Immediate publication on acceptance

- Inclusion in PubMed, CAS, Scopus and Google Scholar

- Research which is freely available for redistribution

Submit your manuscript at www.biomedcentral.com/submit
C Biomed Central 